\title{
Article \\ Recyclable Graphene Sheets as a Growth Template for Crystalline ZnO Nanowires
}

\author{
Yeonhoo Kim ${ }^{1, \dagger}$, Dongheun Kim ${ }^{1, \dagger}{ }^{\dagger}$ Eric Auchter ${ }^{1}$, Justin Marquez ${ }^{1}$, Roxanne Tutchton ${ }^{2} \mathbb{}$, Nan Li ${ }^{1}$, \\ Ting S. Luk ${ }^{3}$, Enkeleda Dervishi ${ }^{4, *}$, Yong-Jin Kim ${ }^{5}$, Jian-Xin Zhu ${ }^{2}$ and Jinkyoung Yoo ${ }^{1, * \mathbb{C}}$ \\ 1 Center for Integrated Nanotechnologies, Los Alamos National Laboratory, Los Alamos, NM 87545, USA; \\ yeonhoo@lanl.gov (Y.K.); heun82@gmail.com (D.K.); elauchter@gmail.com (E.A.); \\ jmarquezche@gmail.com (J.M.); nanli@lanl.gov (N.L.) \\ 2 T-4, Los Alamos National Laboratory, Los Alamos, NM 87545, USA; rtutchton@lanl.gov (R.T.); \\ jxzhu@lanl.gov (J.-X.Z.) \\ 3 Center for Integrated Nanotechnologies, Sandia National Laboratories, Albuquerque, NM 87185, USA \\ tsluk@sandia.gov \\ 4 SIGMA-2, Los Alamos National Laboratory, Los Alamos, NM 87545, USA \\ 5 School of Physics and Astronomy, University of Manchester, Oxford Road, Manchester M13 9PL, UK; \\ dibykim@gmail.com \\ * Correspondence: enkeleda@lanl.gov (E.D.); jyoo@lanl.gov (J.Y.) \\ + These authors contributed equally to this work.
}

Citation: Kim, Y.; Kim, D.; Auchter, E.; Marquez, J.; Tutchton, R.; Li, N.;

Luk, T.S.; Dervishi, E.; Kim, Y.-J.; Zhu, J.-X.; et al. Recyclable Graphene Sheets as a Growth Template for Crystalline ZnO Nanowires. Nanomaterials 2021, 11, 2093. https:// doi.org/10.3390/nano11082093

Academic Editor: Jiye (James) Fang

Received: 30 June 2021

Accepted: 13 August 2021

Published: 18 August 2021

Publisher's Note: MDPI stays neutral with regard to jurisdictional claims in published maps and institutional affiliations.

\begin{abstract}
Recent advances in nanoscience have opened ways of recycling substrates for nanomaterial growth. Novel materials, such as atomically thin materials, are highly desirable for the recycling substrates. In this work, we report recycling of monolayer graphene as a growth template for synthesis of single crystalline $\mathrm{ZnO}$ nanowires. Selective nucleation of $\mathrm{ZnO}$ nanowires on graphene was elucidated by scanning electron microscopy and density functional theory calculation. Growth and subsequent separation of $\mathrm{ZnO}$ nanowires was repeated up to seven times on the same monolayer graphene film. Raman analyses were also performed to investigate the quality of graphene structure along the recycling processes. The chemical robustness of graphene enables the repetitive $\mathrm{ZnO}$ nanowire growth without noticeable degradation of the graphene quality. This work presents a route for graphene as a multifunctional growth template for diverse nanomaterials such as nanocrystals, aligned nanowires, other two-dimensional materials, and semiconductor thin films.
\end{abstract}

Keywords: graphene; ZnO; recycling; hydrothermal synthesis; raman spectroscopy

\section{Introduction}

Epitaxy of thin films and nanomaterials is a crucial step for device manufacturing. However, conventional epitaxy is preferably conducted when original substrates and overgrown materials possess similar lattice constants and thermal expansion coefficients. To utilize the epilayer, the substrates are mostly disposed of or used as a base substrate in devices. Since the cost of substrates for epitaxy process takes up a significant portion in device manufacturing, there has been an intensive research effort to recycle substrates. Epitaxial lift-off (ELO) is a technique of recycling wafers [1], a typical process sequence for ELO is formation of sacrificial layer, epitaxy of desired materials, and post-processing to release the epitaxial thin films and nanomaterials. Although the ELO technique has demonstrated the reuse of substrates for various device applications, such as thin film photovoltaic cells, photodetectors, and light-emitting diodes [1-3], it still requires chemomechanical polishing, which induces substrate material loss of $\sim 10 \mu \mathrm{m} /$ recycle. The material loss results in a process cost increase comparable to $\sim 20 \%$ of the substrate price [4].

Graphene provides novel opportunities for substrate recycling because of its selfterminated surface without surface dangling bonds, which usually act as origins of interfacial defects and strong chemical bonding between graphene and an overgrown ma- 
terial. Successful demonstrations of van der Waals epitaxy and remote epitaxy show that graphene is a versatile substrate for epitaxial growth of elemental and compound semiconductors [5-11]. ELO on graphene can protect the substrate underneath this 2D graphitic structure. However, in this case, the graphene film cannot be recycled because it delaminates from the substrate or it starts forming micro-spalling marks. As of now, large-scale production of high-quality graphene at a low cost is still challenging, given that the use of graphene as a sacrificial layer for the ELO process could lead in a manufacturing cost increase [12]. Although graphene has shown a great potential as a growth template for nanomaterials with optimized morphologies and characteristics, recyclability of graphene nanosheets for repetitive nanomaterial growth has rarely been investigated [13]. Furthermore, $\mathrm{ZnO}$ nanostructures have been studied extensively due to their stable and uniform growth on graphene for various potential applications, such as biomedical and optoelectronic devices $[14,15]$. The growth mechanism of $\mathrm{ZnO}$ nanostructures has also been explored to investigate the influence of the nature of graphene layers [16].

In this work, we report a recycling process of monolayer graphene used as a growth template for synthesis of single crystalline $\mathrm{ZnO}$ nanowires (NWs). The single crystalline $\mathrm{ZnO} N W s$ were selectively grown on the graphene. Moreover, the selective nucleation of $\mathrm{ZnO}$ on graphene was investigated by first-principles calculations based on density functional theory (DFT). Hydrothermal synthesis and delamination processes using chemical exfoliation of the $\mathrm{ZnO}$ wires were repeated on a same graphene layer. Raman spectroscopy analysis demonstrates that the graphene layer remains stable after multiple NW synthesis and delamination processes. In addition, Raman mapping analysis was performed to investigate the quality of graphene surfaces along repetition of $\mathrm{ZnO} \mathrm{NW}$ growth and subsequent delamination. The chemical robustness of graphene enables the reuse of the graphene layer for repeated nanomaterials growth (at least seven times) without noticeable degradation of its quality. This work provides a route for graphene as a recyclable growth template for various materials.

\section{Experimental Details}

Monolayer graphene was synthesized on a $0.1 \mathrm{~mm}$ thick Cu foil (CAS\# 7440-50-8, purchased from Alfa Aesar) using a low-pressure chemical vapor deposition which was previously reported [17]. The $\mathrm{Cu}$ foil was annealed at $950{ }^{\circ} \mathrm{C}$ for $1 \mathrm{~h}$ under $\mathrm{Ar} / \mathrm{H}_{2}$ atmosphere. Next, methane $\left(\mathrm{CH}_{4}, 15 \mathrm{sccm}\right)$ are introduced in the system while the temperature was increased to $1020^{\circ} \mathrm{C}$ for $20 \mathrm{~min}$. Lastly, the sample was rapidly cooled down to room temperature. The synthesized graphene sheet with a surface area of $1.5 \mathrm{~cm} \times 1.5 \mathrm{~cm}$ was transferred onto a $\mathrm{SiO}_{2} / \mathrm{Si}$ substrate using a PMMA-free transfer method [18]. The transfer technique utilizes formvar (polyvinyl formal), a chemically stable and easy to remove polymer, for a rapid, clean and reliable transfer of large-area graphene sheets over a desired substrate.

E-beam lithography (EBL) was employed to define the nucleation sites of $\mathrm{ZnO}$ NWs on the graphene sheet. A 300 nm-thick poly (methyl methacrylate) (PMMA, MicroChem ${ }^{\circledR}$ 495 K C3, Newton, MA, USA) layer was deposited by spin coating. The EBL was performed using the JEOL $6300 \mathrm{FS}$ instrument (JEOL Ltd, Akishima, Tokyo, Japan). The $e$-beam exposed PMMA regions defined as dot patterns were removed by development in methyl isobutyl ketone (MIBK):isopropyl alcohol (IPA) solution. The remaining PMMA layer acted as mask of selective growth of $\mathrm{ZnO}$ NWs on the exposed graphene.

$\mathrm{ZnO} \mathrm{NWs}$ were grown using hydrothermal method on the graphene $/ \mathrm{SiO}_{2} / \mathrm{Si}$ substrate. The monolayer graphene $/ \mathrm{SiO}_{2} / \mathrm{Si}$ template was placed upside-down in a Teflonlined autoclave with the nutrient solution. The nutrient solution was prepared by dissolving zinc nitrate hexahydrate $\left(25 \mathrm{mM}, \mathrm{ZnO}\left(\mathrm{NO}_{3}\right)_{2} \cdot 6 \mathrm{H}_{2} \mathrm{O}\right)$, hexamethylenetetramine $(25 \mathrm{mM}$, $\mathrm{C}_{6} \mathrm{H}_{12} \mathrm{~N}_{4}, \mathrm{HMTA}$ ), and polyethylenimine (5 mM, PEI) in deionized water. Zinc nitrate hexahydrate and HMTA were employed as precursors to form ZnO. PEI was used to induce growth along one principal direction by adsorbing onto nonpolar sidewalls of $\mathrm{ZnO}$ 
crystal. The synthesis was performed at $95^{\circ} \mathrm{C}$ for $4 \mathrm{~h}$. Details of the hydrothermal growth procedure are reported elsewhere [19].

Raman analyses for point spectra and mapping were taken by a homebuilt system, which was calibrated using a Si wafer. A frequency doubled $532 \mathrm{~nm} \mathrm{Nd:YAG} \mathrm{laser} \mathrm{was}$ used to probe the nanostructures and the Raman signal was collected via a liquid nitrogen cooled CCD detector (Princeton Instruments, Inc., Trenton, NJ, USA). For the microphotoluminescence (PL), a frequency quadrupled Nd:YAG laser with the wavelength of $266 \mathrm{~nm}$, a pulse width of $400 \mathrm{ps}$, and a repetition rate of $10 \mathrm{kHz}$ was employed as the excitation source.

\section{Results and Discussions}

The surface morphology and the microstructures of the $\mathrm{ZnO}$ NWs were investigated by scanning electron microscopy (SEM, FEI Quanta 400 F, 10 kV, FEI Company, Hillsboro, OR, USA) and transmission electron microscopy (TEM, FEI Tecnai F20, 200 kV, FEI Company, Hillsboro, OR, USA). Figure 1a shows a selective growth of the ZnO NWs on graphene, while Figure $1 \mathrm{~b}$ presents a tilted-view SEM image of $\mathrm{ZnO}$ NWs grown on graphene. The $\mathrm{ZnO}$ NWs were preferably grown on graphene, while no growth was observed on the $\mathrm{SiO}_{2}$ surface. Position-controlled hydrothermal growth of $\mathrm{ZnO}$ micro/nanostructures on graphene has been reported by several research groups [20-23]. Earlier, $\mathrm{ZnO}$ seed layers were employed to promote the growth of $\mathrm{ZnO}$ structures. However, direct growth of $\mathrm{ZnO}$ NWs on graphene without any seed layer has not been fully understood. Nucleation of $\mathrm{ZnO}$ crystallites by hydrothermal growth is explained by either homogeneous nucleation in a nutrient solution or heterogeneous nucleation on hydrophilic surfaces [19]. Our observation of selective nucleation of $\mathrm{ZnO}$ on graphene layer is incomprehensible by the conventional interpretations, since pristine graphene is not hydrophilic without undergoing additional chemical treatments for introduction of surface functional groups. To elucidate on the observation, the selective nucleation of $\mathrm{ZnO}$ on graphene was investigated by DFT calculations. Figure 1c shows the model systems for the DFT calculations. The super cells consisting of the $\mathrm{ZnO}$ structure bonded to commensurate supercells of $\mathrm{SiO}_{2}$ or graphene were formed to reduce required computational resource and approximate the binding energies between $\mathrm{ZnO}$ and the substrates without consideration of edge-effects. The total energies of supercells of $\mathrm{ZnO}, \mathrm{SiO}_{2}$, and graphene were calculated based on DFT as implemented in the Vienna Ab-initio Simulation Package (VASP). All calculations were conducted with projector augmented-wave (PAW) potentials using the generalized gradient approximation (GGA) of Perdew, Burke, and Ernzerhof (PBE). Each model system was relaxed along the $\mathrm{z}$-direction using preconditioned residual-minimization until the force on the atoms was less than $0.01 \mathrm{eV} / \AA$. In these calculations, no edge or vacuum effects were considered.

The relative binding energy per $\mathrm{Zn}$ atom is defined as

$$
E_{B}=\left(E_{\text {substrate }}+E_{\mathrm{ZnO}}-E_{\mathrm{ZnO} / \text { substrate }}\right) / \mathrm{n},
$$

where $E_{\text {substrate }}$ is the total energy of the substrate, $\mathrm{SiO}_{2}$ or Graphene, $E_{\mathrm{ZnO}}$ is the total energy of the $\mathrm{ZnO}$ nanorod, $E_{\mathrm{ZnO} / \text { substrate }}$ is the total energy of the combined nanorod/substrate system, and $n$ is number of $\mathrm{Zn}$ or $\mathrm{O}$ atoms at the surface of the substrate. The binding energies were calculated to be 0.3654 and $1.7864 \mathrm{eV}$ for the $\mathrm{ZnO} / \mathrm{SiO}_{2}$ and $\mathrm{ZnO} /$ graphene structures, respectively. The higher binding energy for $\mathrm{ZnO} /$ graphene compared to $\mathrm{ZnO} / \mathrm{SiO}_{2}$ indicates that the growth of $\mathrm{ZnO}$ nanostructures is energetically more favorable on graphene than on $\mathrm{SiO}_{2}$ [24]. 

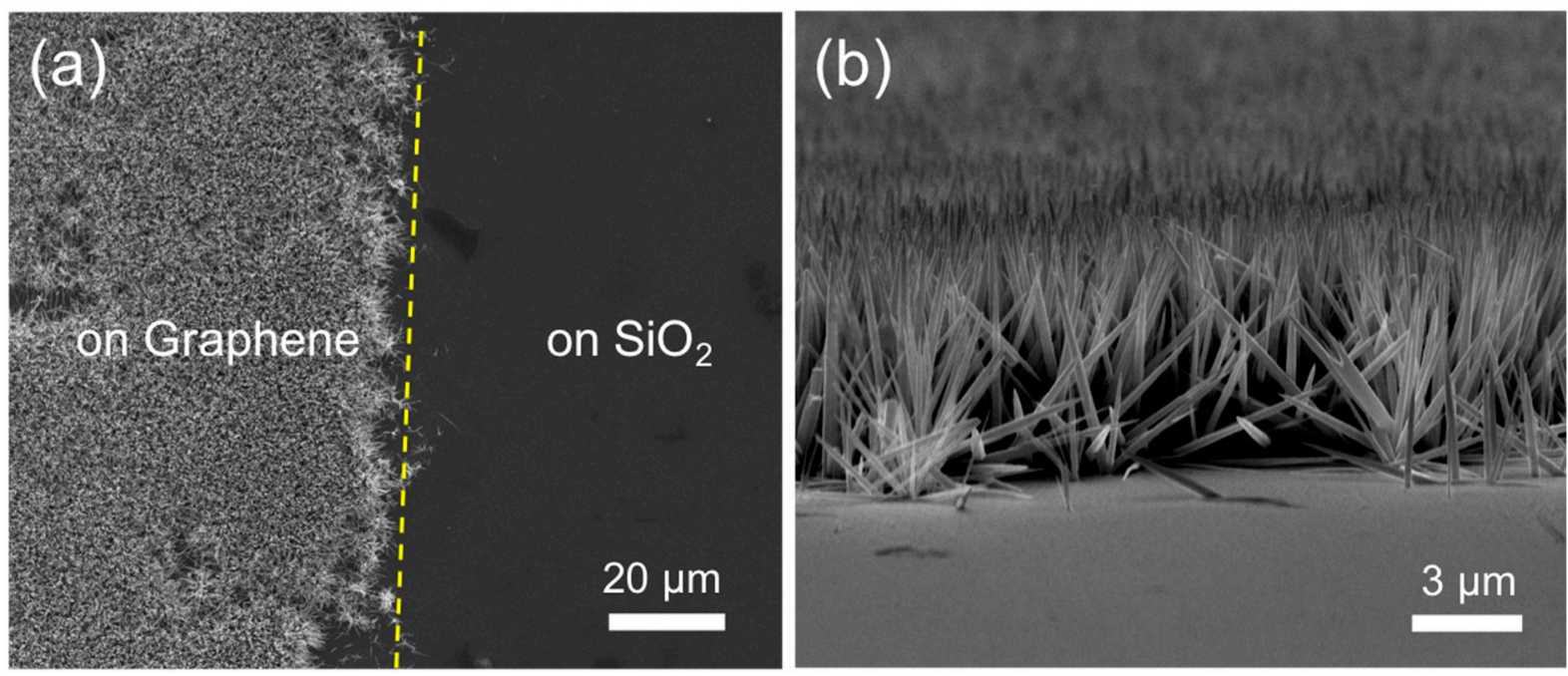

(c)

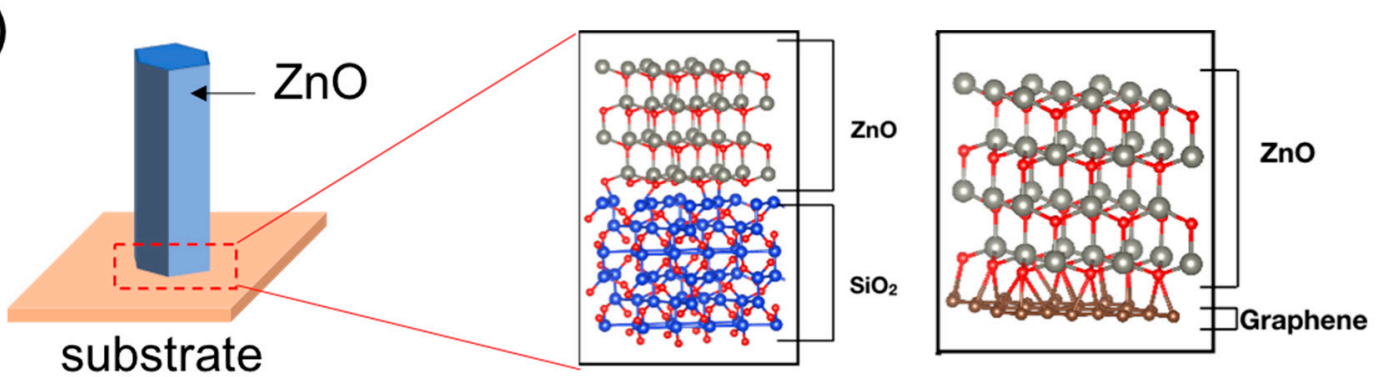

Figure 1. (a) Top-view and (b) tilted-view SEM images of $\mathrm{ZnO}$ wires grown on graphene. (c) The supercells composed of $\mathrm{ZnO}, \mathrm{SiO}_{2}$, and graphene for the DFT calculations.

TEM was used to investigate the structural characteristics of the ZnO NWs on graphene. Figure 2a shows a low magnification TEM image of a $\mathrm{ZnO} \mathrm{NW}$ with a sharp tip. The tapered shape with sharp tips is commonly observed for ZnO NWs prepared by hydrothermal method under alkaline conditions and non-catalyzed growth via metal-organic CVD $[5,15]$. Figure $2 b, c$ indicate that the $c$-oriented $\mathrm{ZnO} \mathrm{NW}$ is single crystalline. The formation mechanism of sharp tips of the $\mathrm{ZnO}$ NWs can be attributed to surface energy anisotropy that (0001) c-plane has the highest surface energy in wurtzite ZnO. Hence, reduction of the surface area of (0001) plane is energetically favorable [25].

The optical properties of the $\mathrm{ZnO}$ NWs were investigated using micro-photoluminescence ( $\mu-\mathrm{PL})$ spectroscopy at room temperature (RT). The strong PL emission centered at $3.25 \mathrm{eV}$ on the graphene, shown in Figure $2 d$, corresponds to the free excitonic emission of $\mathrm{ZnO}$ [26]. Moreover, there was no noticeable emission in visible wavelengths, of which origins are corresponding to oxygen vacancies and zinc interstitials [27]. The luminescence from the $\mathrm{SiO}_{2}$ region was negligible. High crystallinity and dominant free excitonic emission in RT $\mu-P L$ spectrum of the ZnO NWs demonstrate that graphene can be employed as a growth template for high-quality $\mathrm{ZnO}$ nanostructures. 


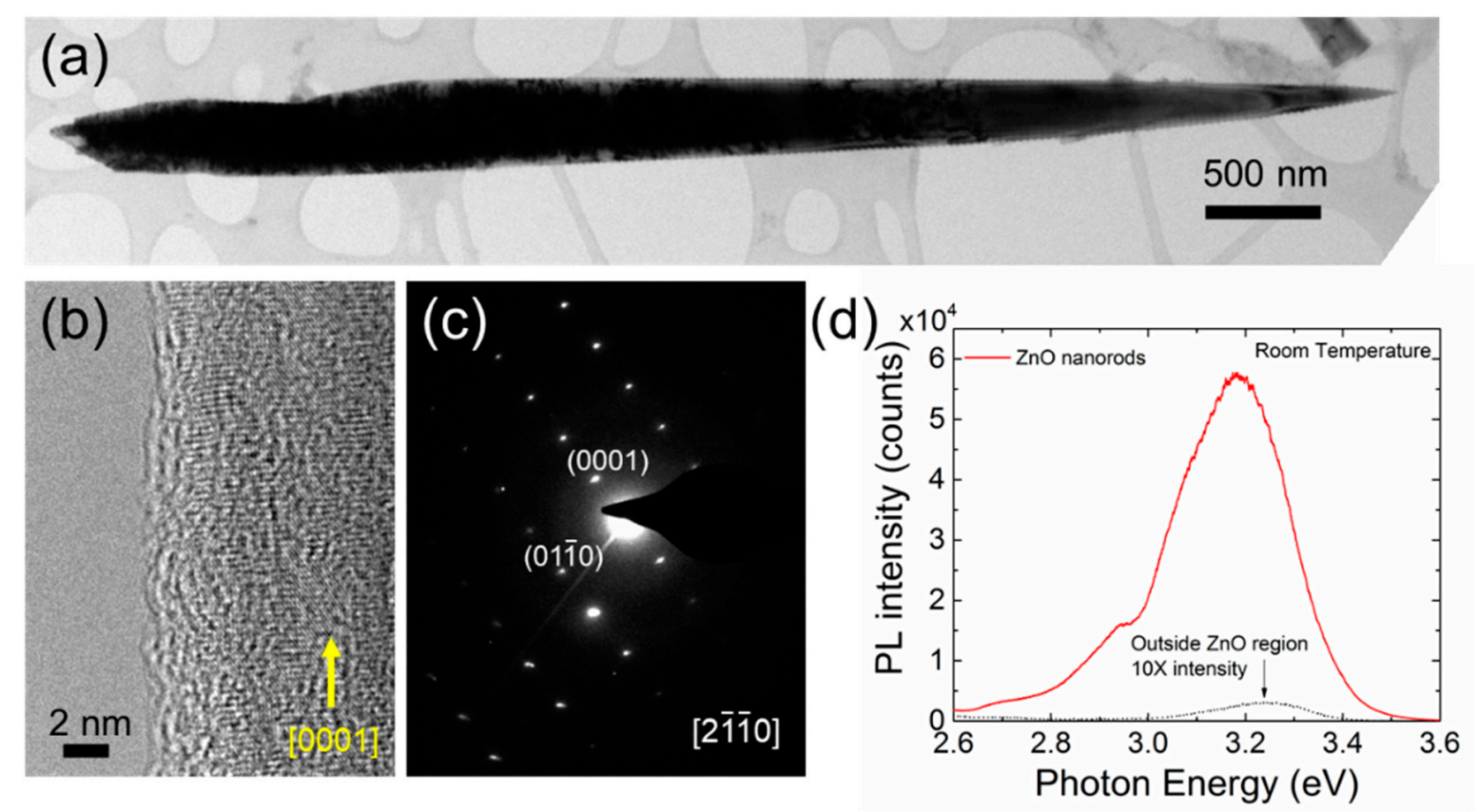

Figure 2. (a) Low magnification, (b) high-resolution TEM images, and (c) electron diffraction pattern of an individual $\mathrm{ZnO}$ NW grown on CVD-graphene via hydrothermal method. (d) Room temperature $\mu-\mathrm{PL}$ spectra of $\mathrm{ZnO}_{\mathrm{NWs}}$ and $\mathrm{SiO}_{2}$ outside graphene.

To recycle graphene layers and repeatedly synthesize $\mathrm{ZnO} N W$ s, repetitive detachment processes of $\mathrm{ZnO}$ NWs are required. Mechanical exfoliation is an alternative way to separate the semiconducting materials from graphene surface. However, the mechanical exfoliation method easily damages either $\mathrm{ZnO}$ or graphene layer. Hence, we developed a wet chemical delamination process to simultaneously preserve the original structure of the $\mathrm{ZnO} N W s$ and that of the graphene layer. The slanted $\mathrm{ZnO}$ NWs (Figure $1 \mathrm{~b}$ ) result in an areal density gradient of the projected NWs region. While the roots of $\mathrm{ZnO} N W s$ are dense, the tips of the NWs are sparse. The ZnO NWs grown on the graphene layer were covered with PMMA via spin coating. PMMA solution (PMMA $950 \mathrm{~K}$ ) was dropped on the $\mathrm{ZnO}$ NWs grown on graphene and baked at $180{ }^{\circ} \mathrm{C}$ on a hot plate for $3 \mathrm{~min}$. To cover the tips of the NWs, the PMMA coating process was repeated three times. Because the radius of gyration of PMMA $950 \mathrm{~K}$ is $26 \mathrm{~nm}$, the PMMA solution cannot permeate into a space narrower than the radius of gyration of $26 \mathrm{~nm}$ [28]. Hence, the bottom parts of the $\mathrm{ZnO}$ NW bundles were not covered with PMMA even after repetition of the PMMA coating. The PMMA/ZnO/graphene sample was immersed in $0.01 \mathrm{M}$ hydrochloric $(\mathrm{HCl})$ acid solution for $24 \mathrm{~h}$ to etch only the bottom parts of the $\mathrm{ZnO}$ NWs where PMMA was not covering. Figure 3 a shows the PMMA coating and subsequent PARTIAL etching process of the $\mathrm{ZnO}$ NWs grown on the graphene layer. Figure $3 \mathrm{~b}$ shows the separated $\mathrm{ZnO} \mathrm{NWs}$ coated with the PMMA after the partial etching of $\mathrm{ZnO}$ with $\mathrm{HCl}$. As the $\mathrm{HCl}$ solution at this particular molarity does not introduce any significant damage on graphene, $\mathrm{SiO}_{2}$, and $\mathrm{Si}$, the repetitive growth and etch process can be successfully utilized to recycle the growth templates. 


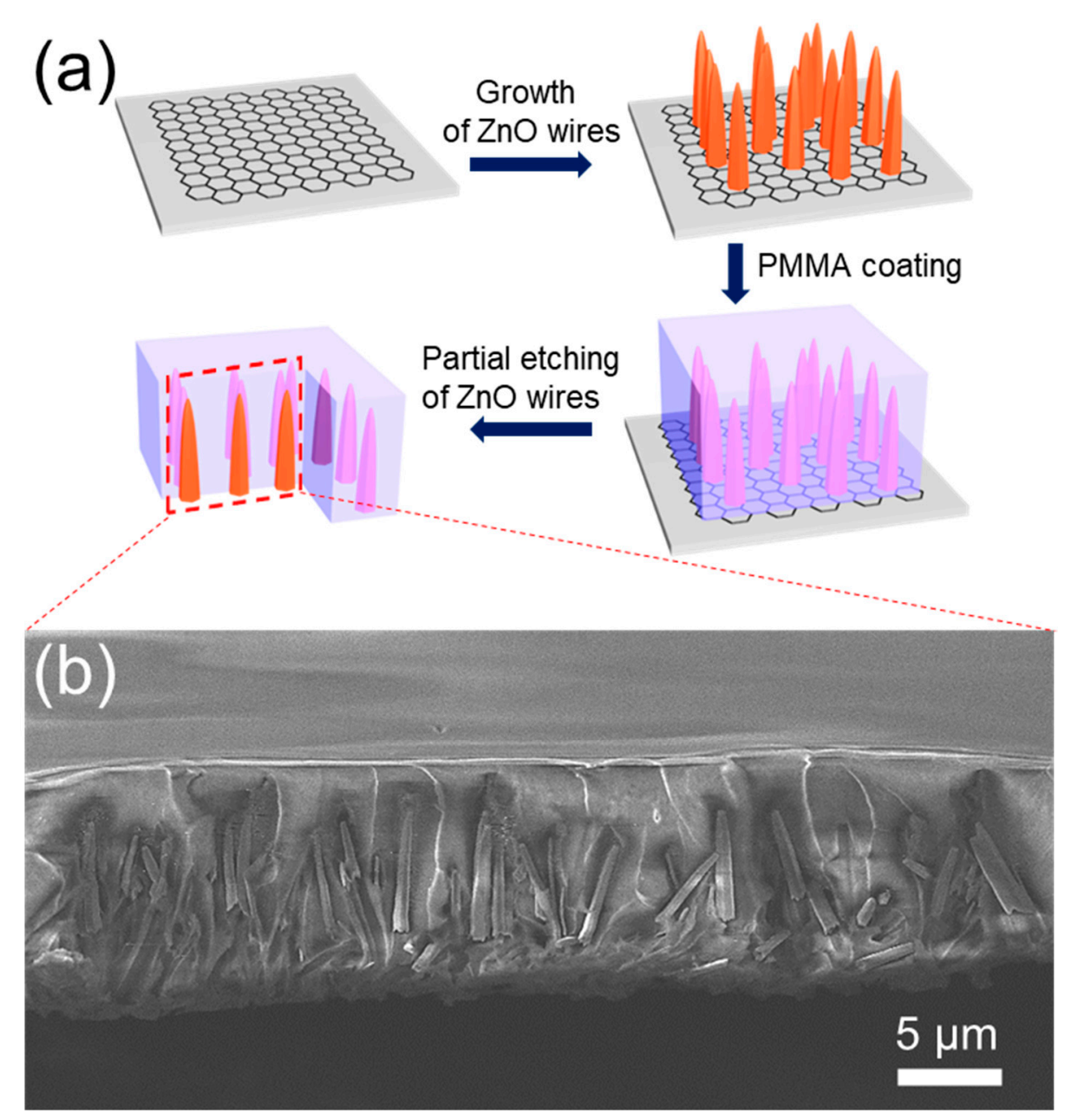

Figure 3. (a) A schematic of the process to separate $\mathrm{ZnO}$ wires from CVD-graphene by PMMA coating and partial wet etching processes. (b) Tilted-view SEM image of $\mathrm{ZnO}$ wires embedded in PMMA after separation from graphene.

\section{Raman Spectroscopy Analyses}

Raman spectroscopy is a non-destructive technique commonly used to probe various carbon nanostructures [29-31]. The position and intensity ratios of the characteristic Raman bands (D, G, and 2D band) of graphene are used to characterize crystallinity, number of layers, doping level, presence of defects and the type of functional groups, etc. A frequency doubled $532 \mathrm{~nm}$ Nd:YAG laser was employed to generate the Raman scattering on the graphene sheets before and after the removal of $\mathrm{ZnO} N W$ s via the wet etching. In order to characterize the same position on the graphene surface, the locations of $\mathrm{ZnO} N W s$ on graphene were defined by $e$-beam lithography. The graphene area was patterned to check the quality of the graphene layer on a defined spot during repetition of the cycle consisting of growth and separation of ZnO NWs. Figure 4a shows the SEM image of hydrothermally grown $\mathrm{ZnO}$ NWs on a hole patterned PMMA/monolayer graphene $/ \mathrm{SiO}_{2} / \mathrm{Si}$ substrate. The observation of selective growth of $\mathrm{ZnO} N W s$ on the exposed graphene areas is consistent with the result shown in Figure 1. The area marked with the 'Red' circle in Figure $4 \mathrm{~b}$ indicates the position where Raman spectra of graphene were acquired before and after growth of $\mathrm{ZnO}$ NWs. Raman spectra were also acquired after growth of $\mathrm{ZnO} \mathrm{NWs}$ and before the partial etching of $\mathrm{ZnO}$ NWs. Figure $4 \mathrm{~b}$ shows three distinct peaks at $\sim 1420$ (D), $\sim 1650$ (G), and $\sim 2740$ (2D) $\mathrm{cm}^{-1}$, which can be assigned to an $\mathrm{A}_{1 \mathrm{~g}}$ breathing mode originating from disorders, to the high frequency $E_{2 g}$, and to the second order of the $\mathrm{D}$, respectively [32]. Raman analyses were employed to monitor band intensities and ratios, hence providing information on graphene structural integrity. Raman spectroscopy is also 
a well-defined method to measure the number of graphene layers [32,33]. The Raman spectrum of the transferred graphene before the $\mathrm{ZnO}$ growth indicates the presence of a crystalline sheet [29]. To maintain the quality of the graphene during transfer, we used our newly developed PMMA-free transfer method while minimizing introduction of defects and avoiding impurities [18,34].

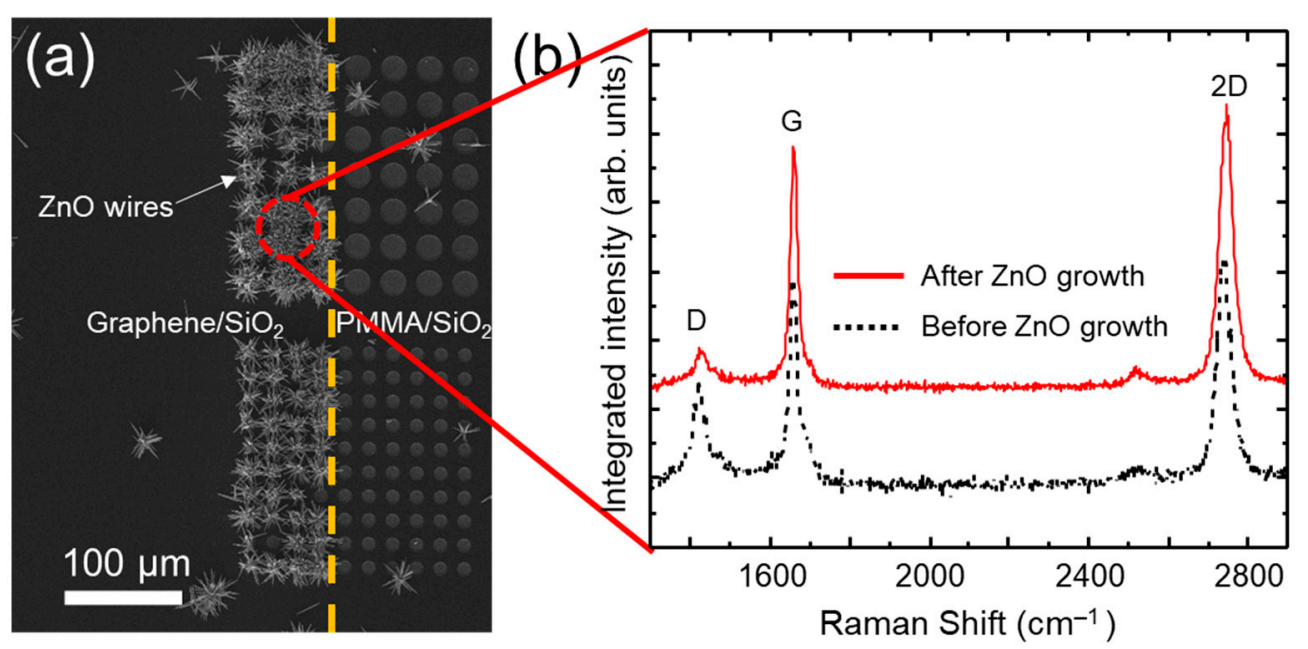

Figure 4. Top-view SEM (a) image of hydrothermally grown ZnO NWs on a hole patterned monolayer graphene $/ \mathrm{SiO}_{2} / \mathrm{Si}$ substrate. The left and the right sides of the orange dashed line are the regions on monolayer graphene $/ \mathrm{SiO}_{2} / \mathrm{Si}$ and $\mathrm{SiO}_{2} / \mathrm{Si}$. (b) The room temperature Raman spectra of the monolayer graphene, obtained at the 'red' circle in (a), before (black, dashed) and after (red, solid) growth of $\mathrm{ZnO} \mathrm{NWs}$.

The Raman spectra shown in Figure $4 \mathrm{~b}$ of the same graphene area before and after $\mathrm{ZnO} N W s$ growth exhibits similar spectral features. Across a series of point scans there was no change in the relative intensities of the $\mathrm{D}, \mathrm{G}$, and 2D peaks, nor was there a consistent shift in peak positions. Additionally, regions of graphene (with and without NWs) probed over a series of 50 scans did not show any significant difference in their corresponding Raman spectra (Figure S1). This was also confirmed by a detailed Raman mapping analysis of these characteristic bands collected in the graphene regions before and after NWs growth (Figure S2). These observations imply that the hydrothermal growth of $\mathrm{ZnO} N W s$ induce neither noticeable incorporation of new disorders in graphene nor alteration of chemical characteristics of graphene. The $\mathrm{ZnO}$ NWs cause some scattering of the beam, but their Raman spectral features are not within the key spectral range of graphene $[35,36]$.

Raman mapping clearly reveals that $\mathrm{ZnO}$ NWs were grown on graphene as shown in Figure 5. Figure 5 exhibits a growth boundary for the NWs, which can be seen along the edge of graphene that bisects the patterned region. Raman intensity mappings ( $G$ and $2 D$ band spectral maps) in Figure 5B show the strong signal response from the graphene where $\mathrm{ZnO} N W s$ were grown. The point spectra in Figure $5 \mathrm{C}$ indicate the graphene presence in the growth regions and the lack of it in other areas.

A key feature of recycling graphene for semiconducting nanomaterial growth is the remarkable properties that this $2 \mathrm{D}$ material continues to possess without degradation when repetitively used as a substrate. Thorough Raman spectroscopy analyses including point spectra and Raman mapping were performed over the growth area to characterize the quality of graphene along the repetition of $\mathrm{ZnO} N W$ s growth and subsequent delamination. The process of growth and delamination of $\mathrm{ZnO}$ NWs was repeated additional seven times on the same graphene. The overlays of all eight spectra of the graphene after each recycling process are shown in Figure 6a. The slight peak shift of $\pm 15 \mathrm{~cm}^{-1}$ and no change in the relative intensities of the three major bands (D, G, 2D) were observed. The slight shift in values of $\pm 15 \mathrm{~cm}^{-1}$ is likely due to environmental or equipment differences on the 
collection days instead of changes to the graphene structure [37]. Raman shifts have been reported to be affected by numerous environmental factors such as temperature, laser power, and excitation energy [38,39].

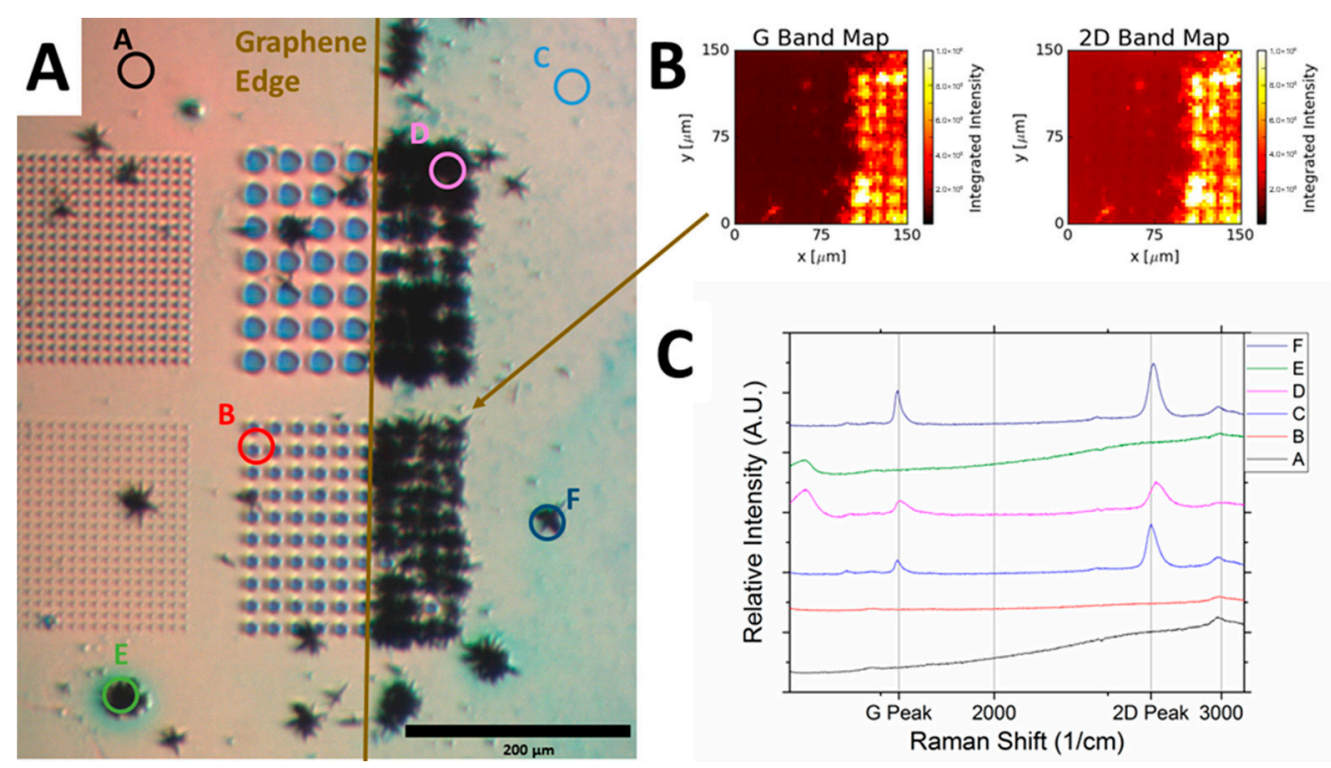

Figure 5. (A) Top-view optical microscopy image of a ZnO NWs/graphene sample with partial coverage of graphene. A-F indicate the locations where Raman measurements were done. (B) Raman intensity maps of $\mathrm{G}$ and $2 \mathrm{D}$ bands confirming graphene areas corresponding to the region of $\mathrm{ZnO}$ NWs growth. (C) Raman spectra collected at the points of A-F.

To further characterize the graphene structure after each growth and removal of nanowires, Raman mapping in a graphene area of $150 \mu \mathrm{m}^{2}$ was conducted. Raman maps of $\mathrm{G}$, and 2D bands in Figure 6b,c indicate no changes in any of the three band intensities after recycling the graphene seven times. The characterization was performed in other areas of graphene, probed after repeated hydrothermal growth and wet etching of $\mathrm{ZnO}$ NWs, yielding similar results (Figure S3). The graphene sheet was not deteriorated even after repeated growth and etching cycles of $\mathrm{ZnO}$ NWs over its surface, making it an attractive substrate for semiconducting nanomaterial growth.

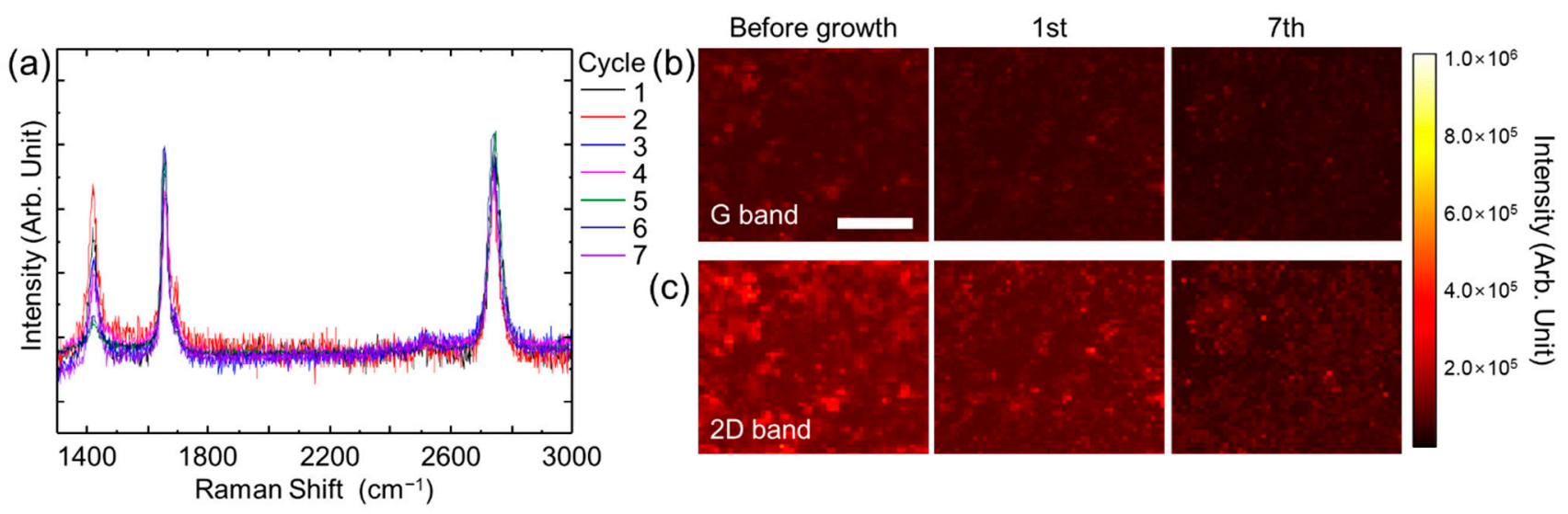

Figure 6. (a) Raman spectra of graphene layer over 7 times of repeated hydrothermal growth and removal of ZnO NWs. (b) Raman map of G and (c) 2D bands before the growth of ZnO NWs and after repetitive growth of ZnO NWs up to 7 times.

Structural and optical properties of the $\mathrm{ZnO}$ NWs grown at the different cycles of the growth-etching process were also characterized. Figure 7a shows the RTPL spectrum 
of $\mathrm{ZnO}$ NWs after the 4th cycle of the recycling process. The weak emission in visible wavelengths indicates low concentration of unintentional defects, such as oxygen vacancies and zinc interstitials. Figure $7 \mathrm{~b}$ exhibits that the $\mathrm{ZnO}$ NWs maintained the identical morphology with same diameter and length after the 7th cycle of the recycling. In addition, $\mathrm{ZnO} N W s$ were not grown on $\mathrm{SiO}_{2}$ layer, as shown in the inset of Figure $7 \mathrm{~b}$, after repetition of the recycling process.

No noticeable degradation of materials quality in both recycled graphene and $\mathrm{ZnO}$ wires region shows that the recycling graphene process in this study is applicable to production of nanomaterials without concern of process-dependent material degradation.
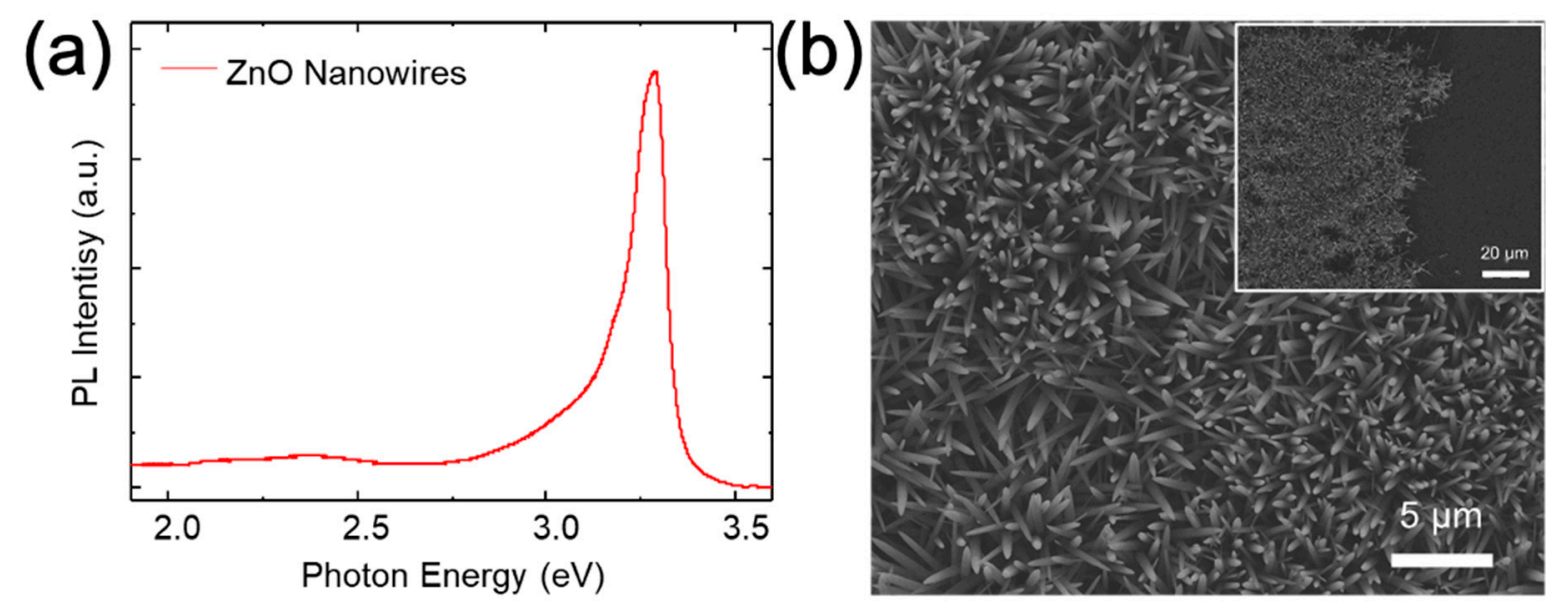

Figure 7. (a) RT PL spectrum of $\mathrm{ZnO}$ wires after the 4th cycle of the growth-etching process. (b) SEM images of ZnO wires grown on graphene after the 7 th growth and etching cycle. The inset is a low magnification image of the $\mathrm{ZnO}$ wires. There was no growth of $\mathrm{ZnO}$ wires on $\mathrm{SiO}_{2}$ layer.

\section{Conclusions}

Recycling of graphene as a growth template for single crystalline ZnO NWs was demonstrated by hydrothermal synthesis and chemical wet etching processes. The crystallinity and position-controlled growth of $\mathrm{ZnO} N W$ s were investigated by the SEM, and TEM analyses and interpreted by theoretical calculations. Raman analyses demonstrate that the graphene template was very stable and reliable after the repetitive growth and detachment processes. Our developed strategy for template-assisted synthesis of nanomaterials based on CVD graphene and recycling of the templates opens up a path forward for this graphitic material as an exciting growth substrate not only the research field, but also manufacturing and industrial fields.

Supplementary Materials: The following are available online at https:/ / www.mdpi.com/article/10 .3390/nano11082093/s1. Additional PL and Raman spectra, Raman mapping data, SEM images are presented in Supplementary Materials.

Author Contributions: Conceptualization, J.Y. and E.D.; Writing—original draft preparation, Y.K. and D.K.; Sample preparation, E.A. and J.M.; Theoretical calculation, R.T.; TEM Analysis, N.L.; Optical analysis, T.S.L.; Data analysis, Y.-J.K. and J.-X.Z.; All authors have read and agreed to the published version of the manuscript.

Funding: This work was performed in part at CINT, a U.S. Department of Energy, Office of Basic Energy Sciences User Facility at Los Alamos National Laboratory (Contract DE-AC52-06NA25396) and Sandia National Laboratories (Contract DE-AC04-94AL85000). The research was financially supported by Laboratory Directed Research and Development program of Los Alamos National Laboratory.

Institutional Review Board Statement: Not applicable.

Informed Consent Statement: Not applicable. 
Data Availability Statement: The data that support the findings of this study are available within this article and its Supplementary Materials.

Acknowledgments: This work was performed, in part, at the Center for Integrated Nanotechnologies, an Office of Science User Facility operated for the U.S. Department of Energy (DOE) Office of Science. Los Alamos National Laboratory, an affirmative action equal opportunity employer, is managed by Triad National Security, LLC for the U.S. Department of Energy's NNSA, under contract 89233218CNA000001. Sandia National Laboratories is a multimission laboratory managed and operated by National Technology \& Engineering Solutions of Sandia, LLC, a wholly owned subsidiary of Honeywell International, Inc., for the U.S. DOE's National Nuclear Security Administration under contract DE-NA-0003525. The views expressed in the article do not necessarily represent the views of the U.S. DOE or the United States Government.

Conflicts of Interest: The authors declare no conflict of interest.

\section{References}

1. Yoon, J.; Jo, S.; Chun, I.S.; Jung, I.; Kim, H.-S.; Meitl, M.; Menard, E.; Li, X.; Coleman, J.J.; Paik, U.; et al. GaAs photovoltaics and optoelectronics using releasable multilayer epitaxial assemblies. Nature 2010, 465, 329. [CrossRef]

2. Kim, Y.; Kim, K.; Jung, S.H.; Kim, C.Z.; Shin, H.-B.; Choi, H.; Kang, H.K. Internal stress-assisted epitaxial lift-off process for flexible thin film (In) GaAs solar cells on metal foil. Appl. Phys. Lett. 2017, 111, 233509. [CrossRef]

3. Youtsey, C.; McCarthy, R.; Reddy, R.; Forghani, K.; Xie, A.; Beam, E.; Wang, J.; Fay, P.; Ciarkowski, T.; Carlson, E.; et al. Wafer-scale epitaxial lift-off of GaN using bandgap-selective photoenhanced wet etching. Phys. Status Solidi B 2017, 254, 1600774. [CrossRef]

4. Woodhouse, M.; Goodrich, A. A Manufacturing Cost Analysis Relevant to Single- and Dual-Junction Photovoltaic Cells Fabricated with III-Vs and III-Vs Grown on Czochralski Silicon; NREL/PR-6A20-601267563; Technical Report; National Renewable Energy Lab. (NREL): Golden, CO, USA, 2013.

5. Kim, Y.-J.; Lee, J.-H.; Yi, G.-C. Vertically aligned ZnO nanostructures grown on graphene layers. Appl. Phys. Lett. 2009, 95, 213101. [CrossRef]

6. Hong, Y.J.; Lee, W.H.; Wu, Y.; Ruoff, R.S.; Fukui, T. van der Waals epitaxy of InAs nanowires vertically aligned on single-layer graphene. Nano Lett. 2012, 12, 1431. [CrossRef] [PubMed]

7. Munshi, A.M.; Dheeraj, D.L.; Fauske, V.T.; Kim, D.-C.; van Helvoort, A.T.J.; Fimland, B.; Weman, H. Vertically aligned GaAs nanowires on graphite and few-layer graphene: Generic model and epitaxial growth. Nano Lett. 2012, 12, 4570. [CrossRef]

8. Kim, J.; Bayram, C.; Park, H.; Cheng, C.-W.; Dimitrakopoulos, C.; Ott, J.A.; Reuter, K.B.; Bedell, S.W.; Sadana, D.K. Principle of direct van der Waals epitaxy of single-crystalline films on epitaxial graphene. Nat. Commun. 2014, 5, 1. [CrossRef]

9. Kumaresan, V.; Largeau, L.; Madouri, A.; Glas, F.; Zhang, H.; Oehler, F.; Cavanna, A.; Babichev, A.; Travers, L.; Gogneau, N. Epitaxy of GaN nanowires on graphene. Nano Lett. 2016, 16, 4895. [CrossRef]

10. Kim, Y.; Cruz, S.S.; Lee, K.; Alawode, B.O.; Choi, C.; Song, Y.; Johnson, J.M.; Heidelberger, C.; Kong, W.; Choi, S. Remote epitaxy through graphene enables two-dimensional material-based layer transfer. Nature 2017, 544, 340. [CrossRef]

11. Jeong, J.; Min, K.-A.; Shin, D.H.; Yang, W.S.; Yoo, J.; Lee, S.W.; Hong, S.; Hong, Y.J. Remote homoepitaxy of ZnO microrods across graphene layers. Nanoscale 2018, 10, 22970. [CrossRef]

12. Lin, L.; Peng, H.; Liu, Z. Synthesis challenges for graphene industry. Nat. Mater. 2019, 18, 520. [CrossRef] [PubMed]

13. Wang, H.; Robinson, J.T.; Diankov, G.; Dai, H. Nanocrystal growth on graphene with various degrees of oxidation. J. Am. Chem. Soc. 2010, 132, 3270-3271. [CrossRef] [PubMed]

14. Kajbafvala, A.; Shayegh, M.R.; Mazloumi, M.; Zanganeh, S.; Lak, A.; Mohajerani, M.S.; Sadrnezhaad, S.K. Nanostructure swordlike $\mathrm{ZnO}$ wires: Rapid synthesis and characterization through a microwave-assisted route. J. Alloy. Compd. 2009, 469, $293-297$. [CrossRef]

15. Zhou, J.; Xu, N.S.; Wang, Z.L. Dissolving behavior and stability of ZnO wires in biofluids: A study on biodegradability and biocompatibility of $\mathrm{ZnO}$ nanostructures. Adv. Mater. 2006, 18, 2432-2435. [CrossRef]

16. Jeong, J.; Jin, D.K.; Cha, J.; Kang, B.K.; Wang, Q.; Choi, J.; Lee, S.W.; Mikhailovskii, Y.V.; Neplokh, V.; Amador-Mendez, N.; et al. Selective-area remote epitaxy of $\mathrm{ZnO}$ microrods using multilayer-monolayer-patterned graphene for transferable and flexible device fabrications. ACS Appl. Nano. Mater. 2020, 3, 8920-8930. [CrossRef]

17. Gao, Y.; Roslyak, O.; Dervishi, E.; Karan, S.N.; Ghosh, Y.; Sheehan, C.J.; Wang, F.; Gupta, G.; Mohite, A.; Dattelbaum, A.M.; et al. Hybrid graphene-giant nanocrystal quantum dot assemblies with highly efficient biexciton emission. Adv. Opt. Mater. 2015, 3, 39-43. [CrossRef]

18. Auchter, E.; Marquez, J.; Yarbro, S.L.; Dervishi, E. A facile alternative technique for large-area graphene transfer via sacrificial polymer. AIP Adv. 2017, 7, 125306. [CrossRef]

19. Kim, Y.-J.; Yoon, A.; Kim, M.; Yi, G.-C.; Liu, C. Hydrothermally grown ZnO nanostructures on few-layer graphene sheets. Nanotechnology 2011, 22, 245603. [CrossRef]

20. Choi, W.M.; Shin, K.-S.; Lee, H.S.; Choi, D.; Kim, K.; Shin, H.-J.; Yoon, S.-M.; Choi, J.-Y.; Kim, S.-W. Selective growth of ZnO nanorods on $\mathrm{SiO}$ 2/Si substrates using a graphene buffer layer. Nano Res. 2011, 4, 440. [CrossRef] 
21. Oh, H.; Park, J.; Choi, W.; Kim, H.; Tchoe, Y.; Agrawal, A.; Yi, G.-C. Vertical ZnO nanotube transistor on a graphene film for flexible inorganic electronics. Small 2018, 14, 1800240. [CrossRef] [PubMed]

22. Kim, Y.-J.; Yoo, H.; Lee, C.-H.; Park, J.B.; Baek, H.; Kim, M.; Yi, G.-C. Position-and morphology-controlled ZnO nanostructures grown on graphene layers. Adv. Mater. 2012, 24, 5565. [CrossRef] [PubMed]

23. Kim, Y.-J.; Yoo, J.; Kwon, B.-H.; Hong, Y.J.; Lee, C.-H.; Yi, G.-C. Position-controlled ZnO nanoflower arrays grown on glass substrates for electron emitter application. Nanotechnology 2008, 19, 315202. [CrossRef] [PubMed]

24. Xu, P.; Tang, Q.; Zhou, Z. Structural and electronic properties of graphene-ZnO interfaces: Dispersion-corrected density functional theory investigations. Nanotechnology 2013, 24, 305401. [CrossRef]

25. Hong, Y.J.; Yoo, J.; Doh, Y.-J.; Kang, S.H.; Kong, K.; Kim, M.; Lee, D.R.; Oh, K.H.; Yi, G.-C. Controlled epitaxial growth modes of $\mathrm{ZnO}$ nanostructures using different substrate crystal planes. J. Mater. Chem. 2009, 19, 941. [CrossRef]

26. Reynolds, D.C.; Look, D.C.; Jogai, B. Optically pumped ultraviolet lasing from ZnO. Solid State Commun. 1996, 99, 873. [CrossRef]

27. Vanheusden, K.; Seager, C.H.; Warren, W.L.; Tallant, D.R.; Voigt, J.A. Mechanisms behind green photoluminescence in ZnO phosphor powders. Appl. Phys. Lett. 1996, 68, 403. [CrossRef]

28. Manfrinato, V.R.; Stein, A.; Zhang, L.; Nam, C.-Y.; Yager, K.G.; Stach, E.A.; Black, C.T. Aberration-corrected electron beam lithography at the one nanometer length scale. Nano Lett. 2017, 17, 4562. [CrossRef]

29. Ferrari, A.C.; Robertson, J. Resonant Raman spectroscopy of disordered, amorphous, and diamondlike carbon. Phys. Rev. B 2001, 64, 075414. [CrossRef]

30. Dervishi, E.; Ji, Z.; Htoon, H.; Sykora, M.; Doorn, S.K. Raman spectroscopy of bottom-up synthesized graphene quantum dots: Size and structure dependence. Nanoscale 2019, 11, 16571. [CrossRef] [PubMed]

31. Dresselhaus, M.S.; Jorio, A.; Hofmann, M.; Dresselhaus, G.; Saito, R. Perspectives on carbon nanotubes and graphene Raman spectroscopy. Nano Lett. 2010, 10, 751. [CrossRef] [PubMed]

32. Ferrari, A.; Meyer, J.C.; Scardaci, V.; Casiraghi, C.; Lazzeri, M.; Mauri, F.; Piscanec, S.; Jiang, D.; Novoselov, K.S.; Roth, S. Raman spectrum of graphene and graphene layers. Phys. Rev. Lett. 2006, 97, 187401. [CrossRef] [PubMed]

33. Graf, D.; Molitor, F.; Ensslin, K.; Stampfer, C.; Jungen, A.; Hierold, C.; Wirtz, L. Spatially resolved Raman spectroscopy of singleand few-layer graphene. Nano Lett. 2007, 7, 238-242. [CrossRef]

34. Auchter, E.; Marquez, J.; Stevens, G.; Silva, R.; Mcculloch, Q.; Guengerich, Q.; Blair, A.; Litchfield, S.; Li, N.; Sheehan, C.; et al. Ultra-thin and strong formvar-based membranes with controlled porosity for micro-and nano-scale systems. Nanotechnology 2018, 29, 215712. [CrossRef] [PubMed]

35. Kumar, S.V.; Huang, N.M.; N Yusoff, N.; Lim, H.N. High performance magnetically separable graphene/zinc oxide nanocomposite. Mater. Lett. 2013, 93, 411. [CrossRef]

36. Zhang, Y.; Ram, M.K.; Stefanakos, E.K.; Goswami, D.Y. Enhanced photocatalytic activity of iron doped zinc oxide nanowires for water decontamination. Surf. Coat. Technol. 2013, 217, 119. [CrossRef]

37. Cançado, L.G.; Jorio, A.; Martins Ferreira, E.H.; Stavale, F.; Achete, C.A.; Capaz, R.B.; Moutinho, M.V.O.; Lombardo, A.; Kulmala, T.S.; Ferrari, A.C. Quantifying Defects in Graphene via Raman Spectroscopy at Different Excitation Energies. Nano Lett. 2011, 11, 3190. [CrossRef]

38. Compaan, A.; Trodahl, H.J. Resonance Raman scattering in Si at elevated temperatures. Phys. Rev. B 1984, 29, 793. [CrossRef]

39. Piscanec, S.; Cantoro, M.; Ferrari, A.C.; Zapien, J.A.; Lifshitz, Y.; Lee, S.T.; Hofmann, S.; Robertson, J. Raman spectroscopy of silicon nanowires. Phys. Rev. B 2003, 68, 241312. [CrossRef] 